\title{
Simultaneous Encoding of Potential Grasping Movements in Macaque Anterior Intraparietal Area
}

\author{
Jason P. Gallivan and Daniel K. Wood \\ Neuroscience Program, University of Western Ontario, London, Ontario N6A 5C2, Canada \\ Review of Baumann et al.
}

The remarkable ability of the visuomotor system to rapidly and flexibly plan goaldirected actions relies on neural mechanisms that remain poorly understood. For instance, we are only beginning to understand how the brain selects one movement plan when many others could also accomplish the same result. Does motor planning rely upon a serial process of developing one ideal movement plan? Or does it rely upon a more parallel approach, with multiple movement plans simultaneously developing and competing against each other for execution? One appealing model of visuomotor planning suggests the latter, with the brain continuously using incoming sensory information to construct neural representations of several potential actions, each of which can be performed in a moment's notice. In this way, the brain begins to specify how to perform actions even before selecting what action it wants to perform (e.g., Cisek, 2007). While some experiments in reach- and eye-related areas of the brain support this hypothesis (e.g., Basso and Wurtz, 1997; Cisek and Kalaska, 2005), in these tasks, the number of possible targets always determined the number of potential movements (reaches or saccades). As such, potential movements were only

Received July 8, 2009; revised Aug. 18, 2009; accepted Aug. 19, 2009.

We are grateful to Jody C. Culham and Melvyn A. Goodale for their comments on the manuscript.

Correspondence should be addressed to Jason P. Gallivan, Department of Neuroscience, University of Western Ontario, London, ON N6A 5C2, Canada. E-mail: jasongallivan@gmail.com.

D0I:10.1523/JNEUROSCI.3245-09.2009

Copyright $\odot 2009$ Society for Neuroscience $\quad$ 0270-6474/09/2912031-02\$15.00/0 represented with respect to locations. New evidence suggests, however, that the parallel specification of multiple potential movements occurs also with respect to a single location.

In a recent study published in The Journal of Neuroscience, Baumann et al. (2009) provide compelling evidence that during grasp planning toward a single object (a handle), neurons in a macaque parietal area involved in hand preshaping simultaneously encode multiple potential grasp movements before one is chosen for action. Although Baumann et al. (2009) noted this finding, their discussion did not emphasize the implications of this result and instead focused on other aspects of the study. The primary purpose of this review is to highlight this valuable finding and show that when it is situated in the context of other relevant studies (not discussed by the authors), it suggests that automatic, simultaneous representations of multiple potential movements occur in several effector-specific regions of the visuomotor system. In this light, our review provides a separate discussion of the Baumann et al. (2009) results and proposes hypotheses to guide future investigations.

A highly influential theory of visuomotor planning, the two-channel hypothesis, proposes that reach-to-grasp actions arise from the combination of separate reach and grasp components, each relying on distinct neuroanatomical substrates: (1) a medial circuit for reaching, comprising the superior parietal lobe (SPL) and dorsal premotor (PMd) area, sensitive to extrinsic target properties like location, and (2) a lateral circuit for grasping, comprising the inferior parietal lobe (IPL) and ventral premotor (PMv) area, sensitive to intrinsic object properties like orientation (Jeannerod et al., 1995). Macaque electrophysiology has identified a region in the IPL, the anterior intraparietal area (AIP), that selectively encodes hand shaping during grasping and shows sensitivity to grasp-relevant object features like size and shape (e.g., Jeannerod et al., 1995). Despite the fact that a single object can afford multiple grip types depending on the intended goal, studies have only examined AIP neural activity in monkeys trained to perform a single grip type to a particular object. While the simplicity of such tasks has largely revealed the functional importance of AIP in grasping, the context and circumstances of everyday situations demand more flexibility in the selection of grip type, and the role of AIP in facilitating such flexibility has remained unexamined.

Baumann et al. (2009) addressed this issue by investigating AIP neural activity during a delayed grasping task in which monkeys were cued to grasp a handle at one of five different orientations using either a precision or a power grasp. When handle orientation and grip type information were concurrently presented, AIP neurons showed sensitivity to handle orientation, grip type, or both and could be classified according to their tuning onset (planning vs movement execution) [Baumann et al. (2009), their Fig. $2 A-C]$. However, in a subset of neurons, when visual presentation of handle orientation and the cues for grip type were sep- 
arated in time (cue separation task), more intriguing properties of the cells emerged. On trials when the handle orientation was revealed before grip type was cued, neurons encoding power or precision grasps were both strongly and equally represented [Baumann et al. (2009), their Fig. $5 B$, Fig. $6 A$, left]. Importantly, however, once grip type information was subsequently cued, the population decreased its firing for the noncued grip type while activity remained constant for the cued grip type. In other words, precision and power grasps were simultaneously encoded before the monkey making a decision between them. This finding supports the notion that the mere presentation of a graspable object can simultaneously activate cell populations representing possible actions (commonly referred to as "affordances") to be performed on the object (for review, see Cisek, 2007).

Consistent with this view, recent evidence from humans and monkeys suggests that reach-related areas within SPL and PMd encode potential actions with respect to object/target locations. A recent fMRI study in humans found that a reachrelated region in the SPL had higher activity for three-dimensional objects within reach than beyond reach, even when participants were not required to act (Gallivan et al., 2009). In other words, when the vicinity of the graspable object introduced the potential for action (i.e., within the operable range of the arm for reaching), SPL was more active. In addition, single-unit recordings have shown that when a monkey is presented with the locations of two potential reach targets for an upcoming arm movement, populations of neurons in PMd simultaneously encode both possible locations (Cisek and Kalaska, 2005). These general findings also receive support from neural recordings in the superior colliculus of macaques, a structure involved in generating eye movements. Basso and Wurtz (1997) show that before the monkey selects a final eye movement, as increasing numbers of potential saccade targets are presented (up to eight), neurons correspondingly represent the increasing number of saccade options. These studies, accompanied by the recent results from Baumann et al. (2009), strongly support the notion that effector-specific areas, before decision, individually encode multiple motor options with respect to an object (possible grip types) and its location (possible reaches and saccades).

In light of the current study, an interesting task for future research will be to test our suspicion that PMv, like AIP [from which it receives heavy projections, e.g., see the study by Jeannerod et al. (1995)], also encodes potential grasp movements during planning. Moreover, given that eye movement areas appear to represent up to at least eight potential saccade targets (Basso and Wurtz, 1997), it remains to be examined how many motor options can be represented in grasp- or reach-specific areas. For example, does an object that affords three or four unique grasps elicit three/four distinct grasp representations in AIP? Interestingly, Baumann et al. (2009) found that some AIP neurons, although showing significant variations in activity throughout the trial, were unmodulated by the tested grip types and object orientations. One interesting possibility is that these neurons may instead show sensitivity to other untested grip types or objects. Electrophysiological recordings and multivoxel pattern analysis of functional magnetic resonance imaging data may provide answers to these questions and shed light on these recently emerging neural properties in effector-specific areas of the brain.

Baumann et al. (2009) also found that the majority of tested neurons in AIP (55\%) represent orientation of the handle immediately upon its presentation, and continue to do so until completion of the grasp movement [Baumann et al. (2009), their Fig. 3; example neurons shown in Fig. $2 A, B$ ]. Interestingly, hand orientation-selective neurons were also recently recorded in area V6A (within the SPL), an area thought to be selectively involved in reaching. Fattori et al. (2009) showed that the majority of recorded neurons (53\%) in V6A were sensitive to both hand orientation and reach location when tested independently during reach-tograsp and reach-to-point tasks, respectively [Fattori et al. (2009), their Fig. 7B]. Until recently, an ongoing debate about the place of object orientation in the "two-visuomotorchannels" hypothesis (i.e., whether it selectively engages the grasp channel) has been addressed primarily by behavioral studies, with evidence showing that target/object orientation affects both reach and grasp kinematics (e.g., Smeets and Brenner, 1999). These two recent electrophysiological findings (Baumann et al., 2009; Fattori et al., 2009) provide new neural evidence to corroborate these behavioral findings, showing that object orientation is encoded within both grasp (AIP) and reach (V6A) parietal areas. This seems consistent with the highly coordinated recruitment of both proximal (reach) and distal (grasp) muscles necessary to orient the wrist (pronate/supinate) for grasping as well as recent anatomical evidence showing connectivity between V6A and AIP (Borra et al., 2008). One hypothesis, given the temporally nonoverlapping components of reach-to-grasp movements (e.g., Smeets and Brenner, 1999), is that object orientation information is first coded within V6A to plan proximal rotations of the arm and then shared with AIP to correspondingly orient the distal hand muscles required for grasping. Another possibility altogether is that these recent findings instead call into question the neuroanatomical segregations implied by the two-visuomotor-channels hypothesisin which case, additional frameworks may be needed to advance our understanding of these issues.

In conclusion, the findings of Baumann et al. (2009) offer unique insights into the neural mechanisms underlying the planning of grasping actions in parietal cortex. In conjunction with other studies, they suggest that each effector-specific area may encode pertinent potential movements before the selection of one for action. This interpretation may provide a useful heuristic to guide future investigations.

\section{References}

Basso MA, Wurtz RH (1997) Modulation of neuronal activity by target uncertainty. $\mathrm{Na}$ ture 389:66-69.

Baumann MA, Fluet MC, Scherberger H (2009) Context-specific grasp movement representation in the macaque anterior intraparietal area. J Neurosci 29:6436-6448.

Borra E, Belmalih A, Calzavara R, Gerbella M, Murata A, Rozzi S, Luppino G (2008) Cortical connections of the macaque anterior intraparietal (AIP) area. Cereb Cortex 18:1094-1111.

Cisek P (2007) Cortical mechanisms of action selection: the affordance competition hypothesis. Philos Trans R Soc Lond B Biol Sci 362:1585-1599.

Cisek P, Kalaska JF (2005) Neural correlates of reaching decisions in dorsal premotor cortex: specification of multiple direction choices and final selection of action. Neuron 45:801-814.

Fattori P, Breveglieri R, Marzocchi N, Filippini D, Bosco A, Galletti C (2009) Hand orientation during reach-to-grasp movements modulates neuronal activity in the medial posterior parietal area V6A. J Neurosci 29:1928-1936.

Gallivan JP, Cavina-Pratesi C, Culham JC (2009) Is that within reach? fMRI reveals that the human superior parieto-occipital cortex encodes objects reachable by the hand. J Neurosci 29:4381-4391.

Jeannerod M, Arbib MA, Rizzolatti G, Sakata H (1995) Grasping objects: the cortical mechanisms of visuomotor transformation. Trends Neurosci 18:314-320.

Smeets JB, Brenner E (1999) A new view on grasping. Motor Control 3:237-271. 